\title{
Noncontact Detection and Analysis of Respiratory Function Using Microwave Doppler Radar
}

\author{
Yee Siong Lee, ${ }^{1}$ Pubudu N. Pathirana, ${ }^{1}$ Robin J. Evans, ${ }^{2}$ and Christopher L. Steinfort ${ }^{3}$ \\ ${ }^{1}$ School of Engineering, Faculty of Science, Engineering and Built Environment, Deakin University, Geelong, VIC 3216, Australia \\ ${ }^{2}$ Department of Electrical and Electronic Engineering, Melbourne University, Parkville, VIC 3010, Australia \\ ${ }^{3}$ University Hospital Geelong, Geelong, VIC 3220, Australia
}

Correspondence should be addressed to Pubudu N. Pathirana; pubudu.pathirana@deakin.edu.au

Received 28 October 2014; Accepted 9 January 2015

Academic Editor: Yu Chen Tsai

Copyright (C) 2015 Yee Siong Lee et al. This is an open access article distributed under the Creative Commons Attribution License, which permits unrestricted use, distribution, and reproduction in any medium, provided the original work is properly cited.

\begin{abstract}
Real-time respiratory measurement with Doppler Radar has an important advantage in the monitoring of certain conditions such as sleep apnoea, sudden infant death syndrome (SIDS), and many other general clinical uses requiring fast nonwearable and noncontact measurement of the respiratory function. In this paper, we demonstrate the feasibility of using Doppler Radar in measuring the basic respiratory frequencies (via fast Fourier transform) for four different types of breathing scenarios: normal breathing, rapid breathing, slow inhalation-fast exhalation, and fast inhalation-slow exhalation conducted in a laboratory environment. A high correlation factor was achieved between the Doppler Radar-based measurements and the conventional measurement device, a respiration strap. We also extended this work from basic signal acquisition to extracting detailed features of breathing function $(I: E$ ratio). This facilitated additional insights into breathing activity and is likely to trigger a number of new applications in respiratory medicine.
\end{abstract}

\section{Introduction}

Respiration monitoring is essential in the diagnosis and treatment of conditions such as chronic obstructive pulmonary disease, heart disease, and a number of sleep related conditions [1]. Furthermore, dysfunctional respiratory patterns such as rapid or shallow breathing [2] or high frequency breathing rates have also been associated with certain psychosomatic conditions [3] all of which, at present, are typically measured via respiration rates alone. However, a more detailed analysis of breathing patterns [4-9] will provide physicians with new insights into diagnostic medicine particularly if this can be performed noninvasively. Noncontact Doppler Radar has already been considered in a variety of patient monitoring and measurement scenarios in healthcare including heartbeat and respiration monitoring in place of conventional methods such as the chest strap, photoplethysmograph [10], and ECG [11]. Research reported using Doppler Radar in measuring human physiological activity [12-18] has predominantly demonstrated the feasibility of Doppler Radar in obtaining breathing frequency or heart rate using FFT, wavelet analysis, or time-frequency analysis $[14,19,20]$.

A complete respiration cycle is typically defined by inhalation (inspiration) and exhalation (expiration) states accompanied by a pause as described in [21]. Breathing rates are predominantly calculated independent of the inhalation to exhalation ratio $(I: E)$ for each breathing cycle. For normal and spontaneous breathing, there is an abundance of time for the exhalation process from the inspired tidal volume, but in certain pathological states, for instance, asthma and COPD (chronic obstructive pulmonary disease), reduced expiratory flow would need longer time to empty the inspired lung volume [22]. Typically, for adults, a normal $I: E$ ratio is in the range of $1: 2$ but this varies between individuals depending on the health and the physiological state of the individual [23]. Consequently more information of each component is extremely important as it can be useful in early detection of several respiratory disorders.

Another important parameter associated with breathing is respiratory tidal volume $[24,25]$ which can also be derived from microwave radar due to the relationship between 
the chest wall displacement and the tidal volume. Different types of breathing can potentially be deduced from such chest wall or abdomen displacement information during inhalation and exhalation. This information can be used to identify different types of breathing signatures such as shallow breathing, deep breathing, slow breathing, fast breathing, and other types of breathing patterns. Indeed, the displacement of the chest wall or abdomen in shallow breathing is expected to be small and the complete breathing cycle would occur in a shorter time period compared to normal breathing.

Doppler Radar operates by transmitting a radio wave signal and receiving the modulated version of the signal due to the motion triggered by the target $[24,26]$. The reflected wave is in the modulated form where it undergoes a frequency shift proportional to the radial velocity that can be described using the Doppler effect. When a target has a quasiperiodic motion, the time varying position of the target can be represented as a phase modulated signal and the phase shift is directly proportional to the object's movement. Thus, the movement of the chest wall/abdomen for respiration due to the inhalation, exhalation, and the pause states can be detected and modelled using the reflected Doppler shifted signal, the main focus of this paper. We provide a comprehensive description of the noncontact respiratory measurement via Doppler Radar which was then validated with independent measurements using a respiration belt and breathing cycle counts. We also demonstrate the different types of inhaling and exhaling states from data collected using our Doppler Radar system. The purpose of this paper is summarized as follows:

(i) investigation of Doppler Radar's feasibility in capturing different types of breathing patterns under various breathing scenarios;

(ii) correction of $I / Q$ signal imbalance and crossvalidation of Doppler breathing signal with standard respiration measurement, the respiration belt (MLT1132 iezo-respiratory belt transducer);

(iii) decomposition of the breathing signal (from Doppler Radar) into its respective inhalation and exhalation components, representing each component model using 4th polynomial fitting (see Table 2(a)) and classifying decomposed breathing components into its respective breathing scenarios.

\section{Methods}

2.1. Respiration Monitoring via Microwave Doppler Radar. The Doppler effect occurs when there is a shift in the frequency of the wave either reflected or radiated, received by an object in motion [27]. Consider a transmitted sine wave signal with an angular frequency $\omega_{0}$,

$$
T_{x}=\sin \left(\omega_{0} t+\phi_{0}\right),
$$

where $T_{x}$ is the transmitted signal, $t$ is the time, and $\phi_{0}$ is the arbitrary phase shift. Assume that the target is stationary at a distance of $r_{0}$ from the radar and the transmission time from radar to target is $r_{0} / c$ where $c$ is the wave propagation velocity.
The target range at time $t$ is given by equation below $r(t)=$ $r_{0}+\dot{r}\left(t-t_{0}\right)$, where $r$ is the range of the target from the radar and $\dot{r}$ (velocity) is the rate of change of $r$ and $t_{0}$ is the time at $r=r_{0}$. The received signal at the stationary target is the same as the transmitted signal at the time $r_{0} / c$ which can be given as

$$
R_{\text {target }}=\sin \left(\omega_{0} t-\frac{\omega_{0} r_{0}}{c}+\phi_{0}\right) .
$$

The received signal from the target at time $t$ would have been sent $\Delta t$ seconds prior to time $t$. This can be represented as $\Delta t=2 r_{0} / c$. Referring to (1), signal can be depicted in the same formulation given as

$$
R_{x}=\sin \left(\omega_{0}(t-\Delta t)+\phi_{0}\right) .
$$

Substituting $\Delta t=2 r_{0} / c$ into (3), the received signal is further represented as

$$
R_{x}=\sin \left(\omega_{0} t-\frac{2 \omega_{0} r_{0}}{c}+\phi_{0}\right) .
$$

For a target moving (radially) with respect to the radar, the distance will vary and by using $r(t)=r_{0}+\dot{r}\left(t-t_{0}\right)$ and $\omega_{d}=$ $2 \omega_{0} \dot{r} / c$, the received signal can be further derived as

$$
\begin{aligned}
R_{x} & =\sin \left(\omega_{0}\left(t-\frac{2 r(t)}{c}\right)+\phi_{0}\right) \\
& =\sin \left(w_{0}\left(t-\frac{2 r_{0}}{c}-\frac{2 \dot{r}\left(t-t_{0}\right)}{c}\right)+\phi_{0}\right) \\
& =\sin \left(\omega_{0}\left(1-\frac{2 \dot{r}}{c}\right) t-\frac{2 \omega_{0}}{c}\left(r_{0}-\dot{r} t_{0}\right)+\phi_{0}\right) \\
& =\sin \left(\left(\omega_{0}-\omega_{d}\right) t-\frac{2 \omega_{0} r_{0}}{c}+\omega_{d} t_{0}+\phi_{0}\right),
\end{aligned}
$$

where the frequency of the reflected signal is shifted by $\omega_{d}$ and the phase angle by $\omega_{d} t_{0}$. Therefore, the Doppler shift $\omega_{d}$ can also be denoted by $\omega_{d}=2 \pi f_{d}$, where $f_{d}=2 \dot{r} f_{0} / c$ is the Doppler shift in Hertz and $f_{0}$ is the transmitted frequency. Using $\lambda=c / f_{0}, f_{d}$ can be written as $f_{d}=-2 \dot{r} / \lambda$ where the negative sign accounts for the fact that if $\dot{r}$ is negative (when the target is approaching), the Doppler frequency will be positive or vice versa [27]. From (5), the phase angle $\Phi$ of the received signal is given as $\omega_{d} t_{0}$. Therefore, the transmitted wave from the radar to the target will be reflected to the receiver with some phase shifting and can be represented as phase modulation given as

$$
\Phi=\frac{2 \omega_{0} \dot{r}}{c} t_{0}=\frac{4 \pi(r)}{\lambda} .
$$

The measurement model for human respiration using Doppler Radar can be derived as follows. Generally, the Doppler shift in frequency is given by

$$
f_{d}(t)=\frac{2 f v(t)}{c}=\frac{2 v(t)}{\lambda},
$$

where $v(t)$ is the velocity of the target, $\lambda$ is the wavelength of the transmitted signal, and $c$ is velocity of the propagating 
wave. Assuming the target to be stationary or undergoing a periodic movement of $x(t)$ with no net velocity, the Doppler frequency shift can be represented in the form of nonlinear phase modulation as the phase signal $\Phi_{r}(t)$ given by $\Phi_{r}(t)=$ $4 \pi x(t) / \lambda$ where $x(t)$ is the displacement of the chest wall or abdomen. Using a continuous wave (CW) radar, the transmitted signal is represented by

$$
T(t)=\cos \left(\omega_{0} t+\phi_{0}(t)\right)
$$

where $T(t)$ is the transmitted signal and $\phi_{0}$ is the arbitrary phase shift or the phase noise of the signal source if the transmitted wave $T(t)$ is reflected by the target/subject at a nominal distance $d_{0}$ with a time varying displacement of $x(t)$ which is caused by the movement of the torso (abdomen). Thus, the distance [28] between the transmitter and the target is given as $d(t)=d_{0}+x(t)$. The measurement of the time delay between the transmitter and the target is denoted as the distance travelled over the signal's propagation velocity given as $d(t) / c$. Thus, due to the movement of the abdomen during the process of respiration, the distance between the antenna and the abdomen at the time of reflection is denoted by $d(t-d(t) / c)$ and the round trip time can be further derived as $t_{d}=2\left(d_{0}+x(t-d(t) / c)\right) / c$.

Using the similar formulation shown in (3) along with $\omega_{0}=2 \pi f$ and $c=f \lambda$, the received signal $R(t)$ can be represented as

$$
\begin{array}{r}
R(t)=\cos \left[\omega_{0}\left(t-t_{d}\right)+\phi\left(t-t_{d}\right)\right] \\
=\cos \left[\omega_{0}\left(t-\frac{2 d_{0}+2 x(t-d(t) / c)}{c}\right)\right. \\
\left.+\phi\left(t-\frac{2 d_{0}+2 x(t-d(t) / c)}{c}\right)\right]
\end{array}
$$

and further approximated as

$$
R(t) \approx \cos \left(2 \pi f t-\frac{4 \pi d_{0}}{\lambda}-\frac{4 \pi x(t)}{\lambda}+\phi\left(t-\frac{2 d_{0}}{c}\right)\right) .
$$

Demodulation of the phase is used to determine the motion signature which can be detected at the receiver. In the direct conversion system, the received signal will be mixed with local oscillator to obtain the baseband output given as

$$
B(t)=\cos \left(\theta+\frac{4 \pi x(t)}{\lambda}+\Delta \phi(t)\right)
$$

In a quadrature receiver system, the received signal will be split into two forms which are an in-phase $\left(I_{B}(t)\right)$ and a quadrature phase $\left(Q_{B}(t)\right)$ signal where the phase difference will be $\pi / 2$. Therefore, general two orthogonal baseband outputs of the quadrature receiver system can be denoted by

$$
\begin{gathered}
I_{B}(t)=\cos \left(\theta+\frac{4 \pi x(t)}{\lambda}+\Delta \phi(t)\right), \\
Q_{B}(t)=\sin \left(\theta+\frac{4 \pi x(t)}{\lambda}+\Delta \phi(t)\right) .
\end{gathered}
$$

Here, $\theta=4 \pi d_{0} / \lambda$ is the constant phase shift dependent on the nominal distance to the target and $\Delta \phi(t)$ is the residual phase noise. The benefit of using a quadrature receiver is to overcome the null problem [11] where at least one output (either $I / Q$ ) is not null when the other is null.

2.2. Signal Processing, Decomposition, and Identification. A complete breathing cycle is comprised of inhalation $(I)$, exhalation $(E)$, and pause components where the ratio of $I: E$ can certainly be asymmetric [23]. Therefore, computation of breathing rates purely based on simple single frequency signatures computed via fast Fourier transforms (FFT) is not sufficient to provide detailed breathing pattern features, particularly for the identification and analysis of respiratory conditions. Firstly, the basic received signal is sent to the I/Q (inphase and quadrature phase) demodulator for direct conversion into its baseband differential $I / Q$ signal and then sampled at $1000 \mathrm{~Hz}$ using NI-DAQ (National Instrument Data Acquisition System). The differential signals were then converted to a single ended baseband signal, removing any DC components of the raw signals, and then processed in two different approaches. In the first approach, the preprocessed raw data was modelled using a piecewise linear least squares approach [29]. In the second approach, the raw data was processed using a SG (Savitzky-Golay polynomial least square) [30] smoothing filter and further analysed using Fourier filtering [31]. The first approach offers a simple method applicable for real-time processing while the second approach offers more accurate identification of the respiration cycle components and their properties, the main focus in this paper.

2.3. Correction of I/Q Amplitude and Phase Imbalance. Two orthogonal outputs ( $I$ and $Q$ ) are obtained from a quadrature receiver system but in practice (due to the imperfection of components in the hardware design), it suffers from amplitude and phase imbalance which affects the accuracy of the recovered data at the output [32]. Consequently, phase and amplitude corrections are necessary to increase accuracy. There are a number of approaches to correct the amplitude and phase imbalance $[33,34]$. In [34], a final form of two orthonormal vectors using a method similar to the Gram Schmidt orthogonalization (GSO) [32] has been proposed as shown in (17). The derivation of this is as follows. The ideally received signal $R_{x}(t)$ is defined by

$$
R_{x}(t)=X_{I} \cos \left(w_{0} t\right)+X_{Q} \sin \left(w_{0} t\right),
$$

where $X_{I}$ and $X_{Q}$ are the in-phase and quadrature phase of the information signal respectively. In our approach, with the presence of amplitude imbalance and phase offset, the received signal at the mixer can be represented as

$$
R_{x}^{\prime}(t)=R_{x}(t) * \cos \left(w_{0} t\right)+R_{x}(t) * A_{e} * \sin \left(w_{0} t+\phi\right),
$$

where $A_{e}$ and $\phi$ are the amplitude and phase imbalance. Demodulation of received signal is as follows:

$$
\begin{gathered}
I^{\prime}=R_{x}(t) * \cos \left(w_{0} t\right), \\
Q^{\prime}=R_{x}(t) * A_{e} * \sin \left(w_{0} t+\phi\right) .
\end{gathered}
$$


Expanding the derivation

$$
\begin{aligned}
I^{\prime}=X_{I} & \cos \left(w_{0} t\right) \cos \left(w_{0} t\right)+X_{\mathrm{Q}} \sin \left(w_{0} t\right) \cos \left(w_{0} t\right), \\
Q^{\prime}= & X_{I} \cos \left(w_{0} t\right) \\
& * A_{e}\left(\sin \left(w_{0} t\right) \cos (\phi)+\cos \left(w_{0} t\right) \sin (\phi)\right) \\
& +X_{\mathrm{Q}} \sin \left(w_{0} t\right) \\
& * A_{e}\left(\sin \left(w_{0} t\right) \cos (\phi)+\cos \left(w_{0} t\right) \sin (\phi)\right) .
\end{aligned}
$$

After the low pass filtering and ignoring the term $1 / 2$, representation of orthogonal $X_{I}$ and $X_{\mathrm{Q}}$ in matrix form

$$
\left[\begin{array}{l}
X_{I} \\
X_{Q}
\end{array}\right]=\left[\begin{array}{cc}
1 & 0 \\
-\tan (\phi) & \frac{1}{A_{e} \cos (\phi)}
\end{array}\right]\left[\begin{array}{c}
I^{\prime} \\
Q^{\prime}
\end{array}\right]
$$

Using (17), correction on amplitude and phase imbalance can be performed. Simulation results of using this approach will be discussed in Section 3.

2.4. The Piecewise Linear Fitting Method. This method fits nonlinear, typically noisy waveforms by choosing an optimal segmentation of the waveform and then fitting each segment with a linear function [29]. Here the segmentation process is critical and, in this case, appropriate lengths of nonoverlapping segments were used. Also, we used fixed nonoverlapping segments of $200 \mathrm{~ms}$ to accommodate the Doppler Radar signal.

2.5. The Savitzky-Golay Method and Fourier Filtering. The Savitzky-Golay filter is a least square polynomial filter [30]. By applying the filter to the noisy data obtained from the chemical spectrum analysers, Savitzky and Golay demonstrated how it reduces noise while preserving the shape and height of waveform peaks. Here, the SG filter was used to smooth the input raw data after the DC components were removed. The output from the SG filter improved the shape of the signal significantly where noise and redundancy were filtered extensively as shown in Figure 3 (data set 1) ((a) and (c)).

The signals were smoothed by SG filter and then reconstructed using Fourier filtering. This was to extract absolute maxima and minima points of the breathing curve that denotes each of the inhalation and exhalation components. Fourier filtering from [31] has already been used as one of the processing algorithms to further eliminate noise and to reconstruct the signals. It is a filtering function that manipulates specific frequency components of a signal by taking the Fourier transform of the corresponding signals which later either attenuate or amplify frequencies of interest. In this paper, the Fourier filter was used to eliminate noise employing a band pass filter depending on the desired breathing frequency range while not distorting the signal significantly. The shape of the Fourier filtered signal was quite similar to the resulting signal from piecewise linear fitting but was smoother and local minima and maxima were prominent.
2.6. Breathing Signal Decomposition. For the breathing cycles obtained from Doppler Radar we assumed that the transition from local minima to local maxima on the curve represents the inhalation component and vice versa for exhalation component, respectively. A peak detection algorithm was then used to determine the maximum and minimum points of each transition defining the inhalation and exhalation components, respectively. These components were extracted separately and represented by a fourth-order polynomial. We then computed the average representation for normal and fast breathing components (inhalation and exhalation) to be used as a model for component identification as discussed in Section 5.3.2.

2.7. Identification-Dynamic Time Warping. Dynamic time warping (DTW) is used to optimally align two time series where one time series is transformed to best fit the other [35]. This technique has been extensively used in speech recognition to identify the similarity of spoken phases from two waveforms as the duration of each spoken sound can vary with similar overall waveform shapes. DTW has also been used in other areas such as data mining and gait recognition [36]. Typically, similarity between two time series for the purpose of classification often requires distance measurement between the two. Computation of Euclidean distance between the two time series may not yield accurate results if one of the two time series is slightly shifted along the time axis. To overcome this limitation, DTW was introduced as described in [35]. Here, we use DTW for registering and comparing breathing components to determine temporal features (extracted breathing component model).

\section{Experiment Mechanism}

Measurement of humans respiration was approved by the Faculty of Science and Technology Ethics Subcommittee HEAG (Faculty Human Ethics Advisory Groups), Deakin University, and all participants provided their written informed consent to participate in this study.

A Doppler Radar system (Figure 1(a)) has a continuous wave $(\mathrm{CW})$ that operates at $2.7 \mathrm{GHz}$ with $2.14 \mathrm{dBm}$, two panel antennae where one is $(\mathrm{Tx})$ and the other $(\mathrm{Rx}), I / Q$ demodulator (Analog Device AD8347), and a data acquisition module (NI-DAQ) were used. The received signals were directly converted into $I / Q$ decomposition using AD8347 where the demodulated signal was then sent to a DAQ for further processing using MATLAB.

For this experiment the subject was positioned $0.5 \mathrm{~m}$ away from the antenna (transmitter, $\mathrm{Tx}$, and receiver, $\mathrm{Rx}$ ). The panel antennae were aligned to focus on the abdomen to capture a better Doppler effect due to respiration. The subject, with normal clothing (see Figure 1(a)) and was asked to stand in front of the antenna and breathe in specific ways for a determined period of time as follows: "normal breathing (maintaining consistency in inhalation and exhalation rate)," "fast breathing (fast inhalation and fast exhalation)," "fast inhalation and slow exhalation," and "slow inhalation and fast exhalation." 


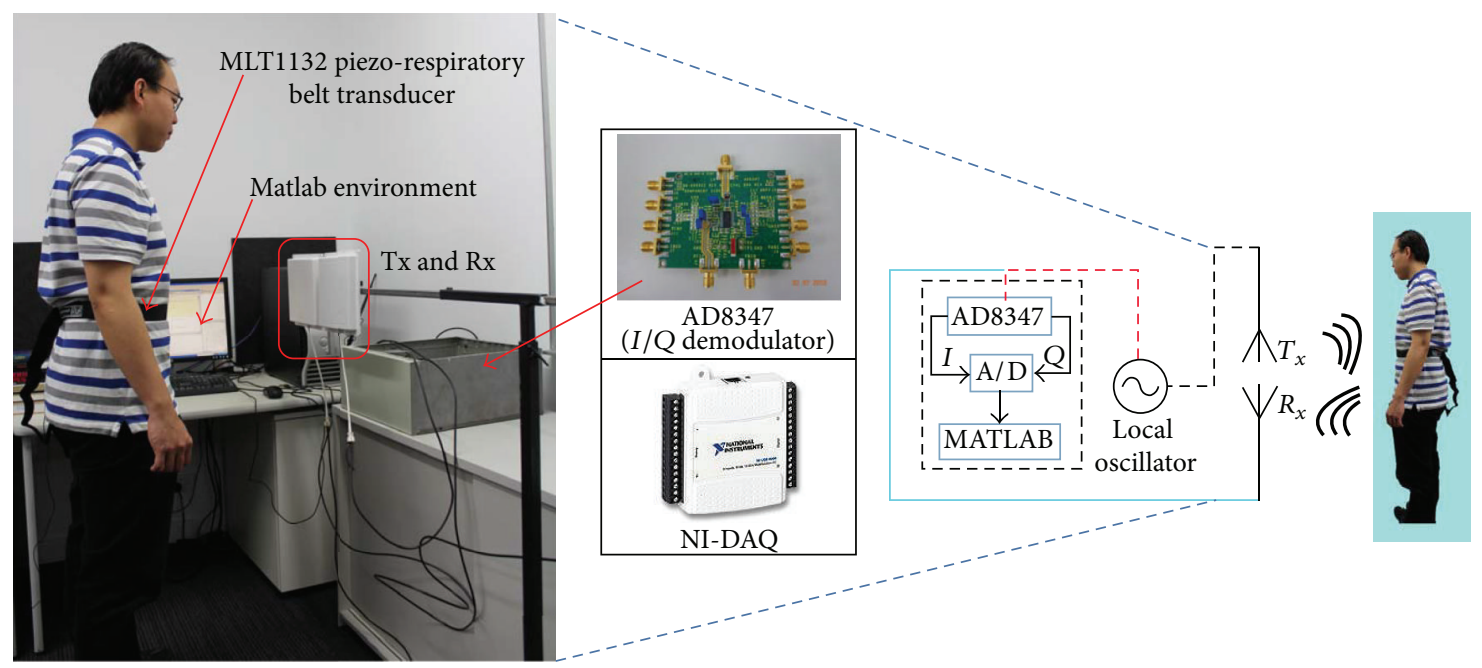

(a) Doppler Radar system

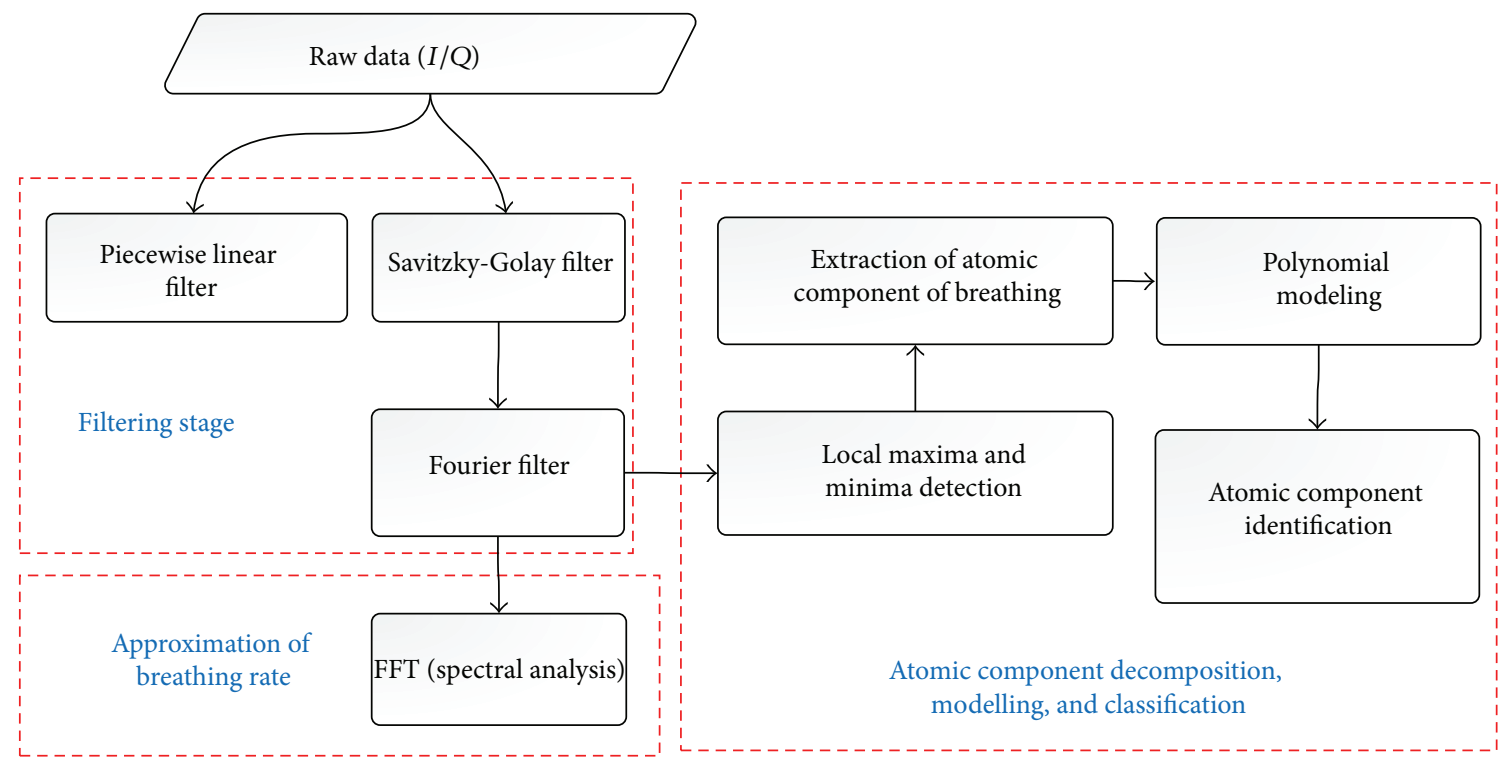

(b) Signal processing flow

FIGURE 1: Doppler Radar system and signal processing flow.

For each breathing pattern, the number of breathing cycles was manually counted and recorded independently to be compared with those computed using the proposed signal processing techniques as shown in Figure 1(b).

For validation purposes, a respiband (MLT1132, piezorespiratory belt transducer) attached to PowerLab (ADInstruments) was used as a reference signal to compare with the Doppler measurements. Results in Figure 2(b) show the normalized raw respiration signal obtained from the respiration belt and normalized filtered Doppler Radar signals.

From (17), the imbalance factors of $A_{e}$ and $\phi$ need to be estimated for $I / Q$ correction. This procedure is similar to the GSO procedure as the quadrature phase signal is orthogonal to the in-phase signal. The simulation was performed by assuming that the breathing frequency is in the vicinity of
$0.2 \mathrm{~Hz}$ in the $I$ and $Q$ representation. In the simulation results shown in Figure $2(\mathrm{a})(\mathrm{C})$, the phase offset of $25^{\circ}$ with amplitude imbalance in quadrature signal was simulated in the noisy signal. We have estimated the amplitude imbalance ratio and phase offset between $I$ and $Q$ signal is corrected the signal using (17) as shown in Figure 2(a). Amplitude imbalance was obtained by taking the average ratio of $Q / I$ while the phase offset was estimated by computing the phase difference between the $I$ and $Q$ signals.

Estimated parameters would be slightly different from the real value due to the noise in the signal but it will be adequate to correct the $Q$ signal based on the $I$ signal. From the results shown in Figure 2(a)(E), the corrected $Q$ signal is similar to the simulated noiseless signal (Figure $2(\mathrm{a})(\mathrm{A})$ ) of the amplitude and the phase offset. The same approach was 


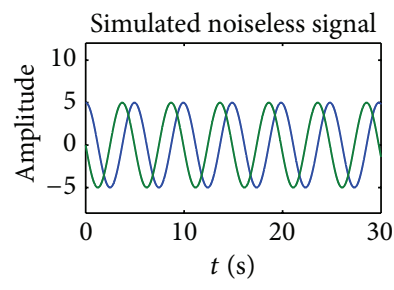

(A)

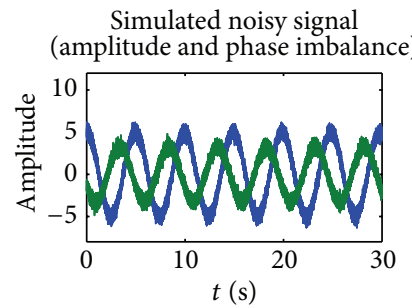

(C)

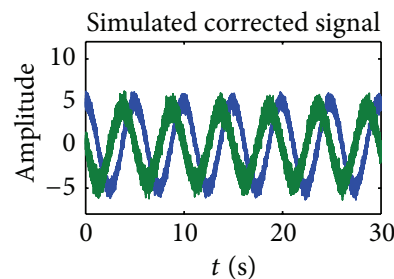

$-I$

$-Q$

(E)

(a) I/Q amplitude and phase imbalance correction simulation

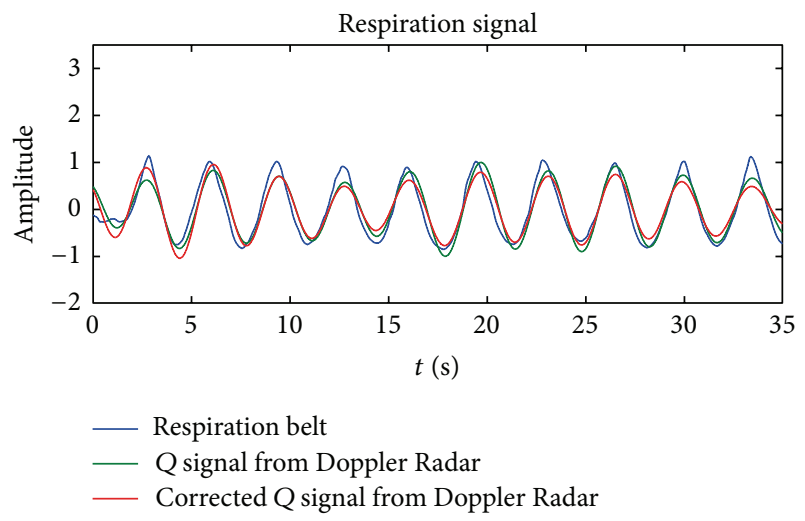

(b) Comparison of respiration belt signal versus Doppler Radar signal

FIGURE 2: I/Q imbalance simulation and results evaluation.

used with the real data and subsequently compared with the respiration belt signal. The corrected $Q$ signal is slightly better than the uncorrected $Q$ signal as the mean squared errors are "0.041651" and "0.050928," respectively (see Figure 2(b)). For further evaluation on the Doppler Radar signals compared to the reference respiration belt, five data sets (a minute of recording for each data set) were collected from the subject (random breathing) where the mean square error (MSE) and correlation coefficient were computed. Results are shown in Table 1 and we notice good correlations obtained between the Doppler signals and the respiratory belt signals.
TABLE 1: Quantitative evaluation of Doppler Radar signal with reference respiration belt.

\begin{tabular}{lcc}
\hline Data set & Mean square error & Correlation coefficient \\
\hline 1 & 0.017 & 0.968 \\
2 & 0.094 & 0.938 \\
3 & 0.009 & 0.965 \\
4 & 0.005 & 0.942 \\
5 & 0.015 & 0.975 \\
\hline
\end{tabular}

TABLE 2: Polynomial modelling and DTW performance evaluation.

(a) Polynomial order evaluation

\begin{tabular}{lcccc}
\hline \multirow{2}{*}{ Order } & \multicolumn{2}{c}{ Inhalation } & \multicolumn{2}{c}{ Exhalation } \\
& RMSE & Corr & RMSE & Corr \\
\hline 1 & $2.14 E-03$ & 0.9912 & $2.04 E-03$ & 0.9918 \\
2 & $2.02 E-03$ & 0.9921 & $2.03 E-03$ & 0.9919 \\
3 & $3.15 E-04$ & 0.9998 & $5.39 E-05$ & 0.9999 \\
4 & $1.97 E-17$ & 1 & $1.02 E-17$ & 1 \\
5 & $3.26 E-17$ & 1 & $1.36 E-17$ & 1 \\
\hline
\end{tabular}

(b) Performance evaluation of random breathing component with selected model

\begin{tabular}{lcccc}
\hline $\begin{array}{l}\text { Breathing } \\
\text { component }\end{array}$ & $\begin{array}{c}\text { Polynomial } \\
\text { model }\end{array}$ & MSE & Corr & Class \\
\hline $\begin{array}{l}\text { Fast } \\
\text { inhalation }\end{array}$ & Normal & $1.11 e-04$ & 0.933 & Fast \\
\hline Normal & Normal & $2.23 e-06$ & 0.999 & Normal \\
inhalation & Fast & $8.37 e-05$ & 0.954 & \\
\hline $\begin{array}{l}\text { Fast } \\
\text { exhalation }\end{array}$ & Normal & $4.58 e-05$ & 0.972 & Fast \\
\hline Normal & Fast & $4.47 e-07$ & 0.999 & \\
exhalation & Normal & $2.50 e-06$ & 0.999 & Normal \\
\hline
\end{tabular}

For the decomposition of the breathing signal into inhalation and exhalation components, it is necessary to calculate the transition time of each breathing component independent of the breathing amplitude. In addition to this, preliminary measurements were obtained from a voluntary subject (asthmatic) to understand if there were any detectable breathing pattern differences in his breathing compared to normal patterns; see Figures 3 and 6 . In the future, as an extension to this current work, more trials will be performed with more subjects, particularly with different breathing conditions for further analysis. This paper is a preliminary exercise to convey the correlation of Doppler Radar with clinically used chest strap devices.

\section{Results}

The results were based on choosing the $I / Q$ baseband signal closest to the optimum point [37] and best matched with 


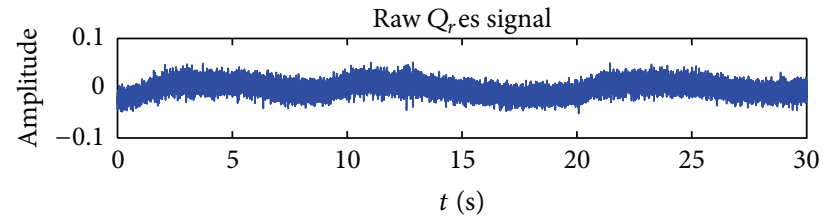

(a)

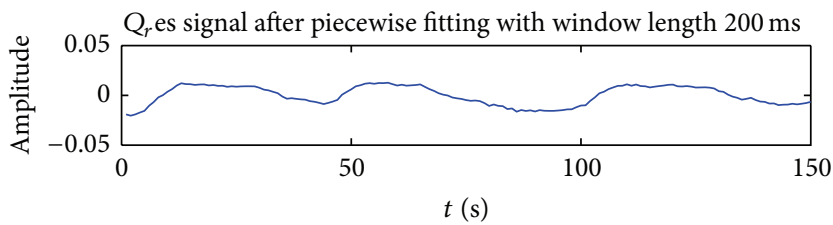

(b)

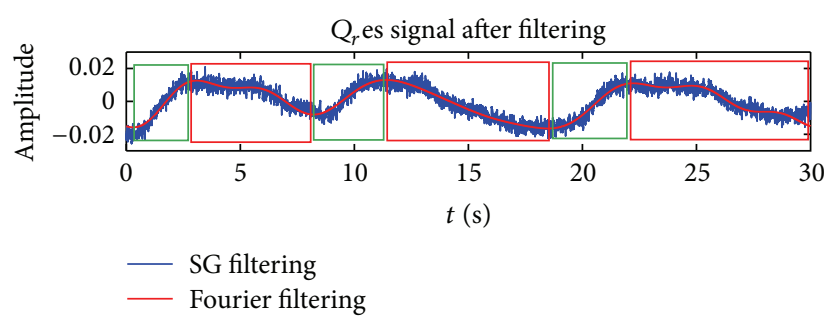

(c)

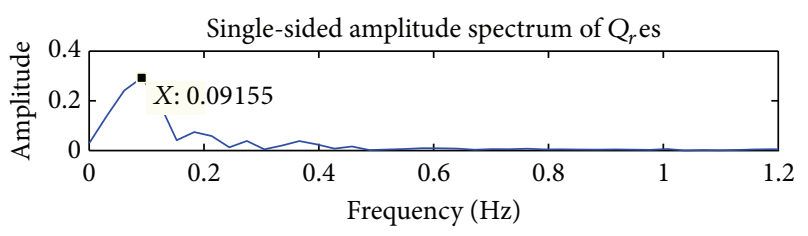

(d)

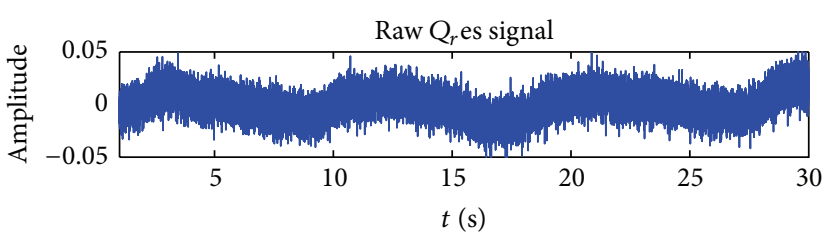

(a)

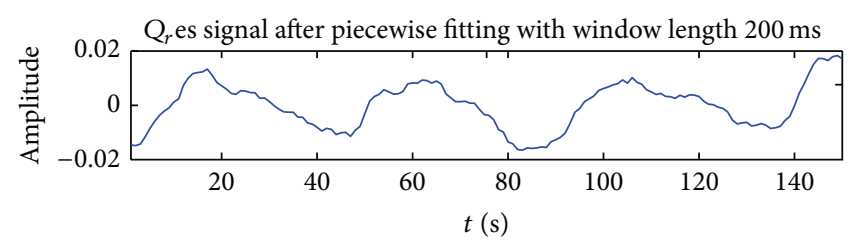

(b)

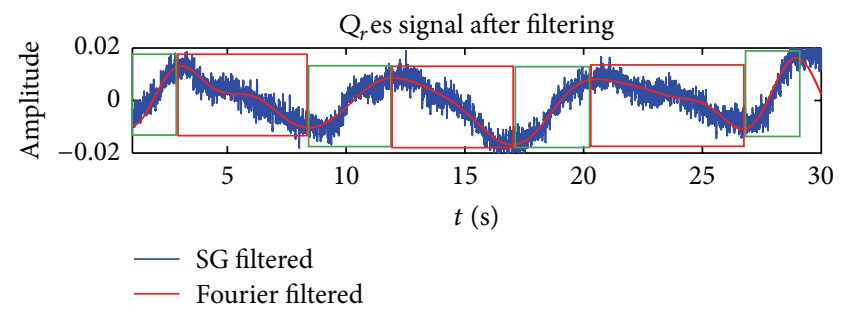

(c)

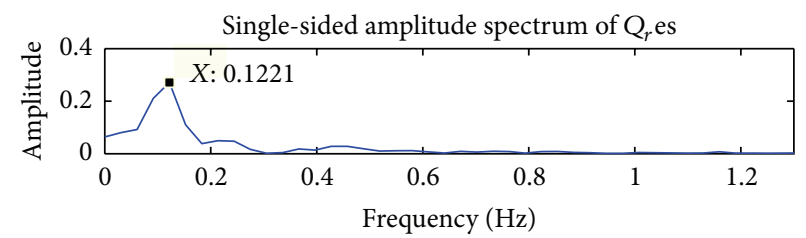

(d)

FIGURE 3: Breathing pattern from voluntary asthmatic subject (data set 1 and data set 2).

the independent breathing measurement. Only a portion of observations were displayed in this paper where the output consisted of Doppler Radar-based measurements for different types of inhalation and exhalation patterns collected over a specific period of time.

4.1. Normal Breathing. Normal adult breathing rates range from 12 to 20 cycles (inhalation, exhalation, and pause) in a minute [4]. Figure 6(a) represents the normal breathing pattern. It can be seen that for a period of 20 seconds there were 5 breaths which corresponded to $0.25 \mathrm{~Hz}(\approx 15$ breaths per minute) and the FFT of the signal shows a constant peak at $0.2441 \mathrm{~Hz}$ or 14.646 breaths per minute. The patterns and extracted rate correlated with the independent breathing counts.

4.2. Fast Breathing. Rapid breathing is typically defined as above 20 breaths per minute for resting adults and this is called Tachypnea [38]. In this experiment, our aim was to establish if breathing at different rates can be detected robustly and the feasibility of subsequent classification.
Figure 6(b) represents the fast breathing pattern with different dynamics. Here, the subject was inhaling and exhaling at a faster rate resulting in a shorter breathing cycle. Results show the occurrence of 12 breathing cycles in a period of 20 seconds (36 per minute). The FFT also shows a peak at $0.6104 \mathrm{~Hz}$ corresponding to 36.6 breaths per minute, similar to the independent breathing cycle counts.

4.3. Slow Inhalation-Fast Exhalation. We mimic another type of breathing scenario where the inhalation is slower than the exhalation rate. Data was collected for a period of 10 seconds and from Figure 6(c), a longer inhalation time (marked in green box) and a shorter exhalation time (marked in red box) are evident. This is as expected as the subject inhales slowly and exhales at a faster pace. Results show that there were two clear breathing cycles in a period of 10 seconds. Observed results show an average of $2.5: 1$ for the $I: E$ ratio where the FFT computation approximated the breathing rate to be $14.65(0.2441 \mathrm{~Hz})$ breaths per minute and the expected breathing rate was 12 breaths per minute from independent measurements. For these particular experiments, an average 
of 2.5 seconds was required for inhalation compared to the one second needed for exhalation.

4.4. Fast Inhalation-Slow Exhalation. Figure 6(d) shows the signal representation for fast inhalation and slow exhalation. Measurements clearly show that two breathing cycles with an average of $1: 2.5 I: E$ ratio occurred. The breathing rate was expected to be 12 breaths per minute and from the FFT, the breathing rate was estimated as $14.65(0.2441 \mathrm{~Hz})$ breaths per minute. Results from both observations clearly show that the exhalation is longer than inhalation. Both the cases discussed in Sections 4.3 and 4.4 further prove that the respiration rate alone is not adequate in describing the respiratory activities of the subjects. A more descriptive information could be obtained through the breathing cycle decomposition approach from the noncontact Doppler Radar measurement.

\section{Discussions}

Results in Section 4 have demonstrated the feasibility of Doppler Radar in capturing various types of breathing dynamics and this section further discusses the importance of breathing cycle analysis, decomposition, and identification.

5.1. Possible Abnormal Breathing Patterns. It is clear that simply recording breathing frequencies, measured as a angular frequency using spectral methods, is inadequate for analysing asymmetric breathing patterns [23], albeit useful for extracting the fundamental cycle for breathing periods. The evidence so far is that decomposing the breathing cycle into its inhalation and exhalation components offers a more accurate and insightful approach to detecting and interpreting breathing and can be performed reliably using Doppler Radar. In this particular experiment, the breathing pattern of a voluntary subject (age: 23, height: $180 \mathrm{~cm}$, and weight: $95 \mathrm{~kg}$ ) who has asthma was collected within the duration of 30 seconds but not during an asthma attack. Results are shown in Figure 3. Notice the inhalation component (marked in the green colour box) is of a shorter duration compared to the exhalation component (marked in the red color box) where the approximated $I: E$ ratio for that subject is $1: 2.5$. Both the results showed a longer duration recorded for exhalation compared to inhalation where the implications are such that the subject could be having difficulties in exhaling [39] and this enforces the value in the analysis by decomposition.

In future work, experiments from Sections 4.1-5.1 will be extended with an increased number of subjects (normal and abnormal) in a clinical trial to further support the qualitative and quantitative evaluations. This can facilitate finding a more accurate and insightful way to describe the respiratory functions using a noncontact form of measurements. Furthermore, additional analysis could be performed, including the amplitude variation and the shape of each decomposed breathing component pertaining to different types of subjects. For instance, amplitude variation in the voluntary subject with asthma was observed to be lesser than that of the subject with normal breathing. Consideration on respiratory effort, breathing patterns, and other related factors (e.g., respiratory function such as tidal volume) would be an essential study in the future in evaluating the potential use of Doppler Radar in respiratory research which includes sensing, detections, analysis, and qualitative assertions.

5.2. Breathing Component Decomposition. Although a complete breathing cycle comprises of inhalation and exhalation, short and even long pauses can also exist between these states depending on the regularity of breathing and other factors such as the need for oxygen, surrounding environment, and so forth. A long pause, for instance, of more than 10 seconds [40] is defined as an abnormal event and is known as apnoea relevant for detecting sleep apnoea and even SIDS. Breathing patterns can also potentially be used together with the analysis of tidal volume [24] to diagnose other aspects of breathing problems such as shallow breathing and the capability in detecting apnoea. These have been reported in [15] using microwave Doppler Radar.

The main purpose of decomposing the breathing cycles is to gain useful information of the breathing activity. For instance, an abnormal breathing rate of 8 breaths/min could be analysed with more information such as inhalation and exhalation rates, and so forth. This can be particularly useful when it could be used in the early diagnosis of specific breathing conditions or in a pulmonary rehabilitation [4143], especially if it could be performed in a noncontact form.

Each of the inhalation and the exhalation components was extracted to obtain the polynomial coefficients from normal and fast breathing data, respectively, and results indicate that a fourth-order RMSE (root mean square error) and Corr (correlation coefficient) polynomial were sufficient to fit these components (e.g., randomly chosen inhalation and exhalation component) as shown in the Table 2(a). Subsequently, using the same approach, the computed fourth-order polynomial model was used to characterise two different types of inhalation and exhalation breathing components (normal and fast). This model was then used to identify the experimental breathing scenario as discussed in Section 5.3.2.

\subsection{Analysis of the Breathing Component}

5.3.1. I: E Ratio Analysis. The ratio between the inhalation or exhalation components was computed from the average time duration in considerations of the entire set. Using the collected data, there were 15 fast and 7 normal components extracted from the data sets and the ratios of each of the components (in comparison with the average time of respective inhalation/exhalation components) are shown in Figure 4. It was seen that there were two distinct groups corresponding to two different breathing dynamics in two different events where this could not be estimated from the respiration rate estimation (spectral analysis).

\subsubsection{Dynamic Time Warping and Evaluation by Correlation.} The time duration for complete inhalation and exhalation components varies between individuals and situations. Therefore, in order to summarise, characterise, compare, and 

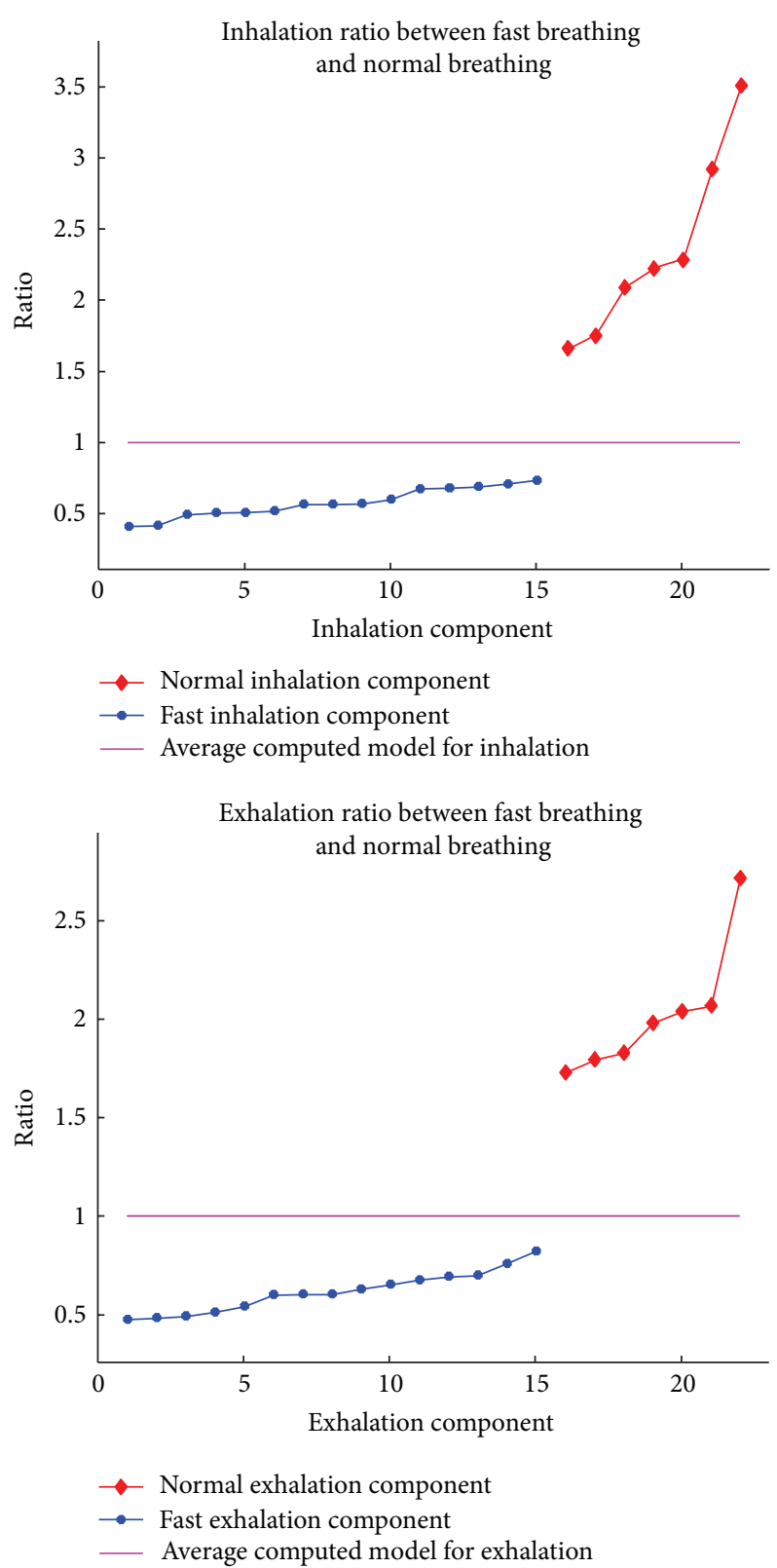

FIGURE 4: Ratio of breathing component.

interpret breathing patterns, a number of alternatives can be considered. In our experiments, these include

(i) extraction of inhalation and exhalation components based on normal and fast breathing criteria;

(ii) computation of fourth-order polynomials model for each breathing condition (normal and fast) from the extracted components, respectively;

(iii) using dynamic time warping to find the optimal alignment between the predefined model from (ii) and the randomly picked breathing component; (iv) using the correlation method to identify the similarity of the aligned results from (iii) for identification and computing the MSE between the curves.

Two different polynomials for inhalation and exhalation in normal and fast breathing were modelled from the data sets (procedure: (i)-(ii)). For validation, dynamic time warping was performed between randomly chosen components (any data set) with the model based on polynomial representation (procedure: (iii)-(iv)).

The purpose of performing this experiment was to use the derived model as a reference and to classify each breathing component based on two different classes. In brief, by deriving a model based on the rate of breathing, we can, in fact, 


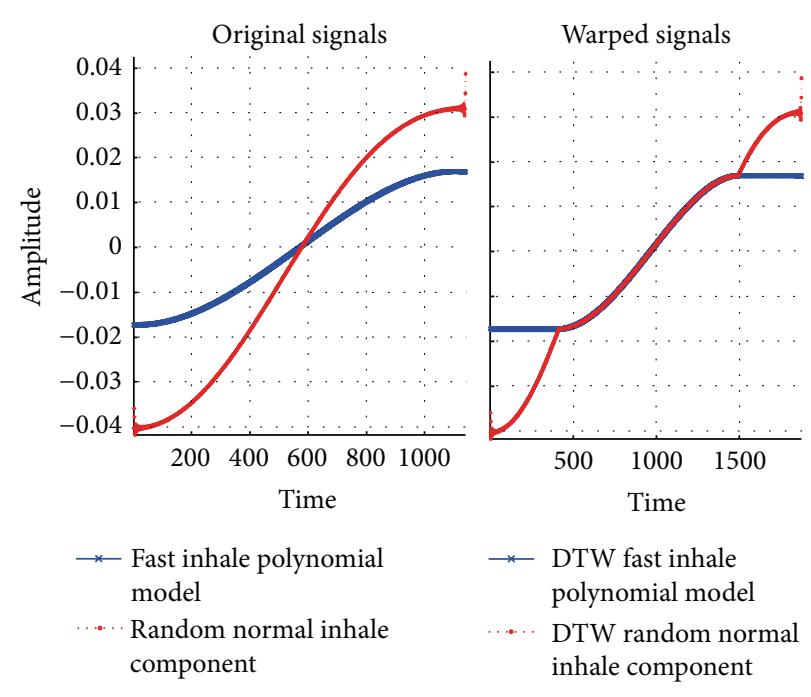

(A) Normal inhale component with fast inhale model
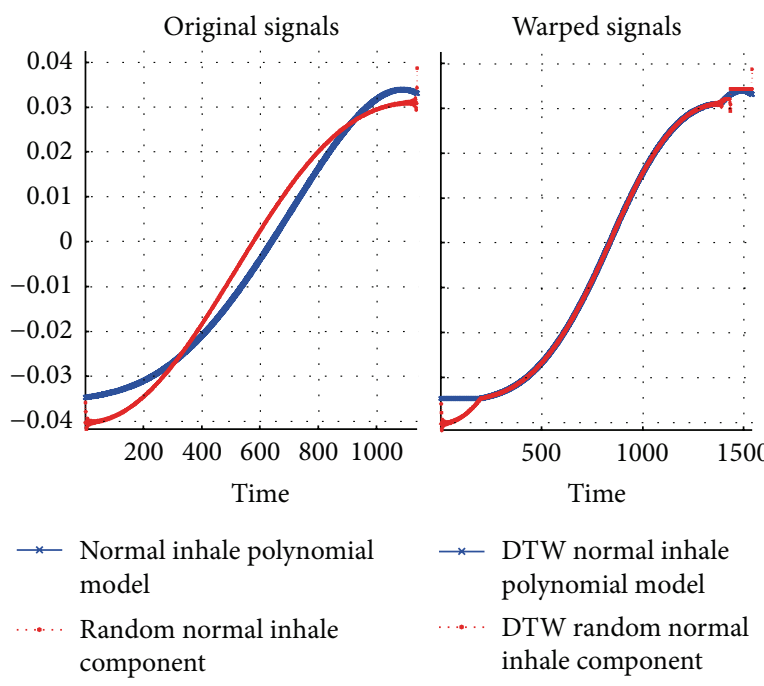

* DTW normal inhale polynomial model

.... DTW random normal inhale component

(a) DTW of normal inhalation component with respective inhalation model

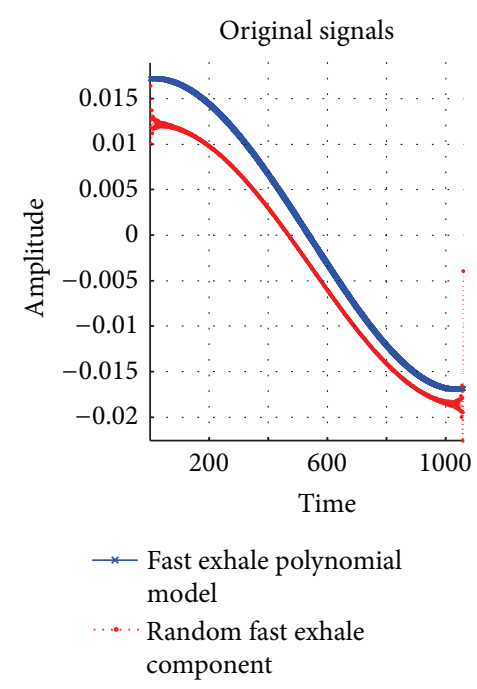

(A) Fast exhale component with fast exhale model
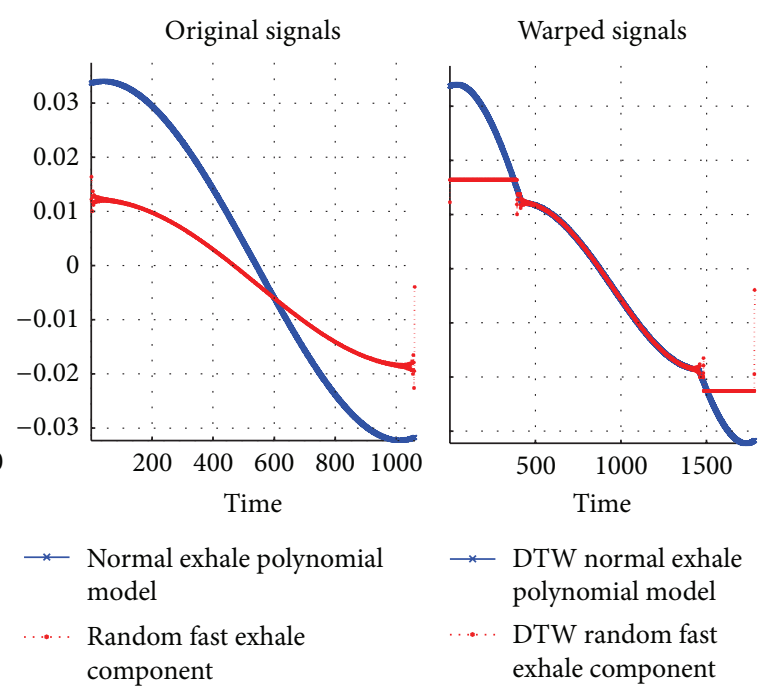

(b) DTW of fast exhalation component with respective exhalation model

Figure 5: DTW evaluation.

identify and correlate the extracted breathing components with the derived model to distinguish different respiratory classes. For validation purposes, the experiments were performed as follows:

(a) fast inhalation component with normal and fast inhalation model;

(b) normal inhalation component with normal and fast inhalation model;

(c) fast exhalation component with normal and fast exhalation model;

(d) normal exhalation component with normal and fast exhalation model.
Each of the breathing components was randomly picked from the data sets. It was then evaluated and represented in terms of mean square error (MSE) and correlation coefficient (Corr) as shown in Table 2(b). For graphical representation, as an example, we associate "normal inhalation component with normal and fast inhalation model" and "fast exhalation component with normal and fast exhalation model" and the results were shown in "Figures 5(a) and 5(b)," respectively.

\section{Conclusions}

In this paper, we have demonstrated the feasibility of breathing detection under varying conditions using Doppler Radar. We have shown that noninvasive breathing detection using 


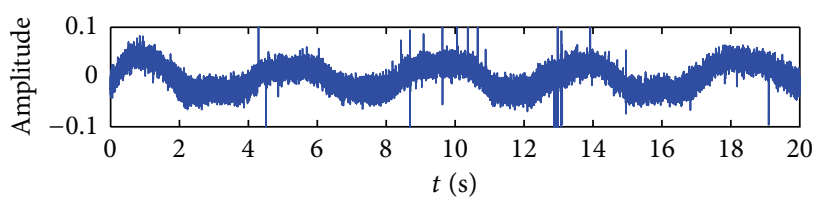

(A) Raw $Q_{r}$ es signal

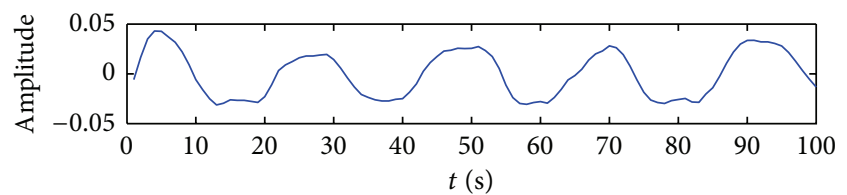

(B) $Q_{r}$ es signal after piecewise fitting with window length $200 \mathrm{~ms}$

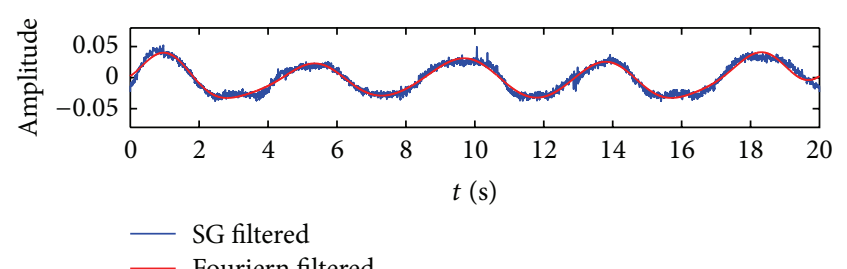

(C) $Q_{r}$ es signal after filtering

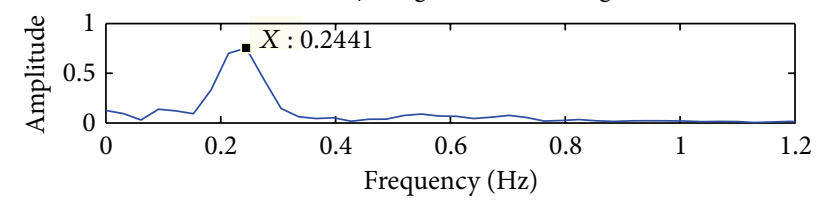

(D) Single-sided amplitude spectrum of $Q_{r}$ es

(a) Normal breathing

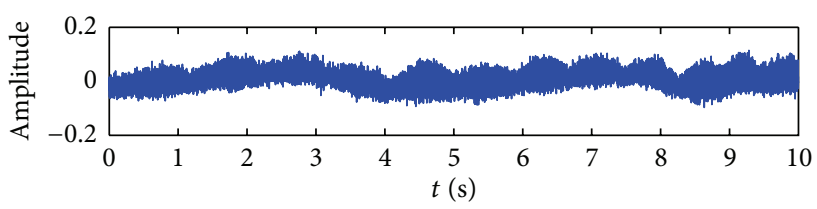

(A) Raw $I_{r}$ es signal

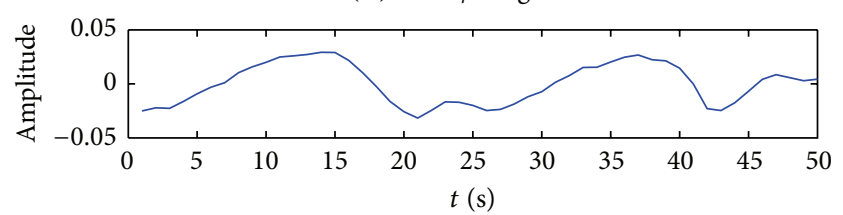

(B) $I_{r}$ es signal after piece wise fitting with window length $200 \mathrm{~ms}$

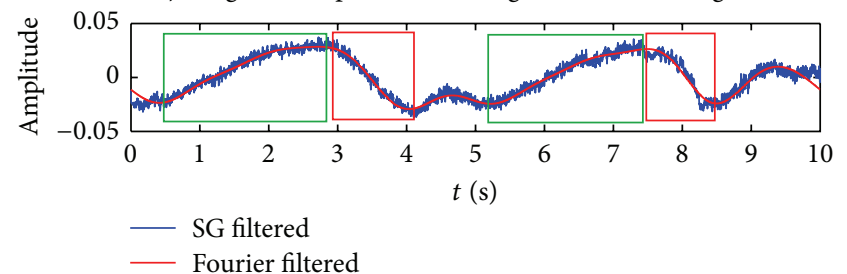

(C) $I_{r}$ es signal after filtering

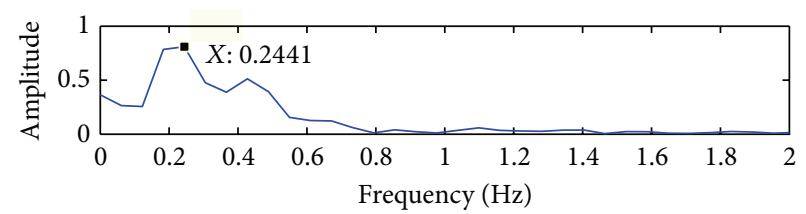

(D) Single-sided amplitude spectrum of $I_{r}$ es

(c) Slow inhalation-fast exhalation

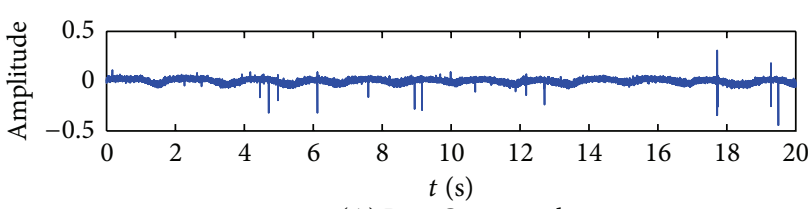

(A) Raw $Q_{r}$ es signal

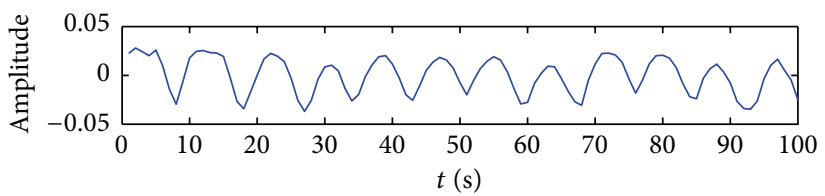

(B) $Q_{r}$ es signal after piecewise fitting with window length $200 \mathrm{~ms}$

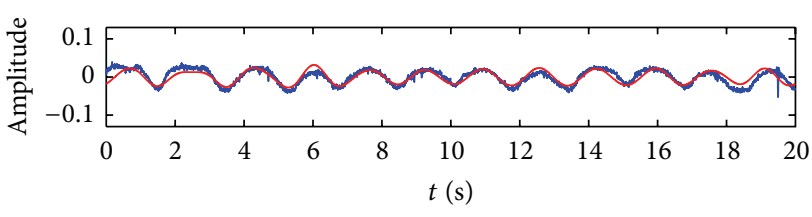

— SG filtered

— Fourier filtered

(C) $Q_{r}$ es signal after filtering

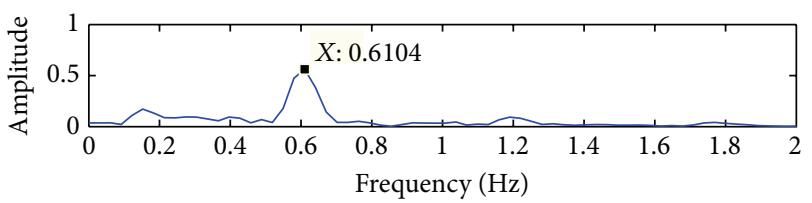

(D) Single-sided amplitude spectrum of $Q_{r}$ es

(b) Fast breathing

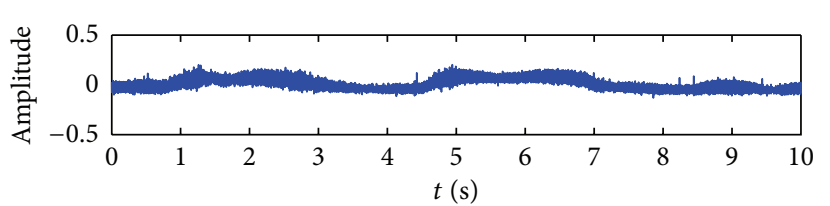

(A) Raw $I_{r}$ es signal

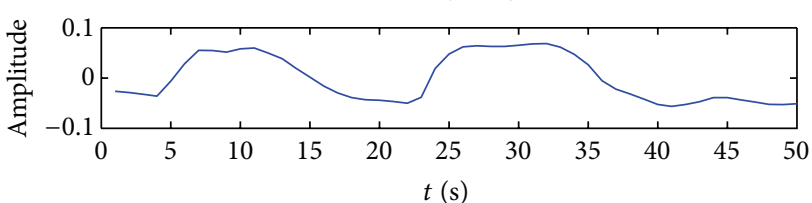

(B) $I_{r}$ es signal after piece wise fitting with window length $200 \mathrm{~ms}$

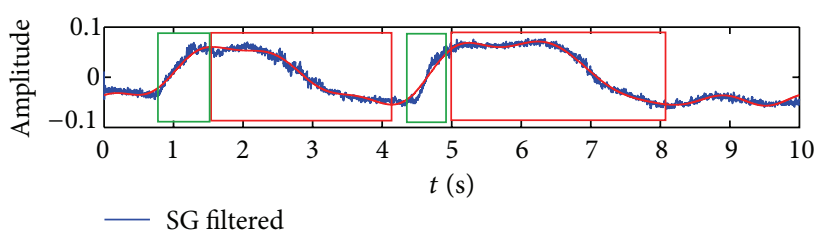

— SG filtered

- Fourier filtered

(C) $I_{r}$ es signal after filtering

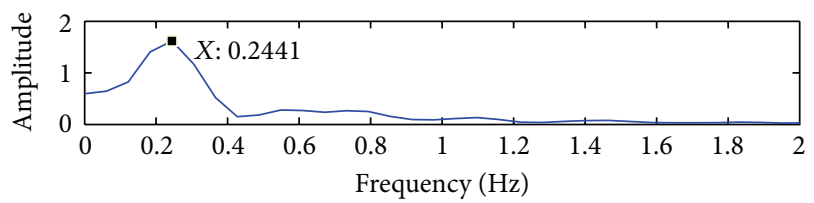

(D) Single-sided amplitude spectrum of $I_{r}$ es

(d) Fast inhalation-slow exhalation

FIGURE 6: Doppler Radar signals from various type of breathing scenarios. 
Doppler Radar could potentially be used to detect different types of breathing patterns such as rapid breathing and slow breathing. We have also demonstrated that by decomposing the respiratory cycle into inhalation, pause, and exhalation, it is possible to extract additional information on the breathing activities. For this purpose, we proposed a fourth-order polynomial to represent each atomic component of breathing and demonstrated the use of DTW in classifying breathing component independently into the corresponding class. In the derived model, each component is associated to a specific breathing scenario, which in particular is fast and normal breathing. Regarding future work, experimental trials will be extended with more subjects as well as improved signal processing techniques (e.g., isolation of motion artefacts and more robust model based filtering techniques), breathing component modelling, and classification techniques.

\section{Conflict of Interests}

The authors declare that there is no conflict of interests regarding the publication of this paper.

\section{Acknowledgments}

This work was supported by Australian Federal and Victoria State Governments and the Australian Research Council through the ICT Centre of Excellence program, National ICT Australia (NICTA).

\section{References}

[1] J. Boyle, N. Bidargaddi, A. Sarela, and M. Karunanithi, "Automatic detection of respiration rate from ambulatory singlelead ECG," IEEE Transactions on Information Technology in Biomedicine, vol. 13, no. 6, pp. 890-896, 2009.

[2] H. Gibson, "A form of behaviour therapy for some states diagnosed as affective disorder," Behaviour Research and Therapy, vol. 16, no. 3, pp. 191-195, 1978.

[3] P. Grossman, "Respiration, stress, and cardiovascular function," Psychophysiology, vol. 20, no. 3, pp. 284-300, 1983.

[4] G. Yuan, N. A. Drost, and R. A. McIvor, "Respiratory rate and breathing pattern," McMaster University Medical Journal, vol. 10, pp. 23-28, 2013.

[5] S. Mondini and C. Guilleminault, "Abnormal breathing patterns during sleep in diabetes," Annals of Neurology, vol. 17, no. 4, pp. 391-395, 1985.

[6] H. Corning, Mosby's PDQ for Respiratory Care-Revised Reprint, Elsevier Health Sciences, 2012, http://books.google.com .au/books?id=hYgfvCdwa3sC.

[7] Y. Munjal, S. Sharma, M. A. K. Agarwal, and P. Gupta, Api Textbook of Medicine, Series G: Reference, Information and Interdisciplinary Subjects Series, Jaypee Brothers, Medical Publishers, 2012, http://books.google.com.au/books?id=L7pW3yGjj7kC.

[8] L. Stead and S. Thomas, Emergency Medicine, Board Review Series, Lippincott \& Williams, 2000, http://books.google.com.au/ books?id=lmTpnSGEYwwC.

[9] B. Aehlert and R. Vroman, Paramedic Practice Today: Above and Beyond, vol. 2, Jones \& Bartlett Learning, 2011, http://books .google.com.au/books?id=gA3mcImmXbAC.
[10] K. Nakajima, T. Tamura, and H. Miike, "Monitoring of heart and respiratory rates by photoplethysmography using a digital filtering technique," Medical Engineering and Physics, vol. 18, no. 5, pp. 365-372, 1996.

[11] D. Girbau, A. Lázaro, Á. Ramos, and R. Villarino, "Remote sensing of vital signs using a doppler radar and diversity to overcome null detection," IEEE Sensors Journal, vol. 12, no. 3, pp. 512-518, 2012.

[12] J. H. Oum, D.-W. Kim, and S. Hong, "Two frequency radar sensor for non-contact vital signal monitor," in Proceedings of the IEEE MTT-S International Microwave Symposium Digest (MTT '08), pp. 919-922, June 2008.

[13] W. Xu, C. Gu, C. Li, and M. Sarrafzadeh, "Robust Doppler radar demodulation via compressed sensing," Electronics Letters, vol. 48, no. 22, pp. 1428-1430, 2012.

[14] N. Birsan, D.-P. Munteanu, G. Iubu, and T. Niculescu, “Timefrequency analysis in Doppler radar for noncontact cardiopulmonary monitoring," in Proceedings of the E-Health and Bioengineering Conference (EHB '11), pp. 1-4, November 2011.

[15] Y. S. Lee, P. N. Pathirana, T. Caelli, and S. Li, "Further applications of Doppler radar for non-contact respiratory assessment," in Proceedings of the 35th Annual International Conference of the IEEE Engineering in Medicine and Biology Society (EMBC '13), pp. 3833-3836, Osaka, Japan, July 2013.

[16] Y. S. Lee, P. N. Pathirana, T. Caelli, and R. Evans, "Doppler radar in respiratory monitoring: detection and analysis," in Proceedings of the 2nd International Conference on Control, Automation and Information Sciences (ICCAIS '13), pp. 224-228, November 2013.

[17] S. Suzuki, T. Matsui, H. Kawahara et al., "A non-contact vital sign monitoring system for ambulances using dual-frequency microwave radars," Medical and Biological Engineering and Computing, vol. 47, no. 1, pp. 101-105, 2009.

[18] S. Suzuki, T. Matsui, H. Imuta et al., "A novel autonomic activation measurement method for stress monitoring: Noncontact measurement of heart rate variability using a compact microwave radar," Medical and Biological Engineering and Computing, vol. 46, no. 7, pp. 709-714, 2008.

[19] O. Boric-Lubecke, V. M. Lubecke, A. Host-Madsen, D. Samardzija, and K. Cheung, "Doppler radar sensing of multiple subjects in single and multiple antenna systems," in Proceedings of the 7th International Conference on Telecommunications in Moderm Satellite, Cable and Broadcasting Services (TELSIKS '05), vol. 1, pp. 7-11, September 2005.

[20] A. Tariq and H. Ghafouri-Shiraz, "Vital signs detection using doppler radar and continuous wavelet transform," in Proceedings of the 5th European Conference on Antennas and Propagation (EUCAP '11), pp. 285-288, April 2011.

[21] A. Abushakra, M. Faezipour, and A. Abumunshar, "Efficient frequency-based classification of respiratory movements," in Proceedings of the IEEE International Conference on Electro/Information Technology (EIT '12), pp. 1-5, May 2012.

[22] D. G. E. Criner and J. Gerard, Critical Care Study Guide, Springer, New York, NY, USA, 2002.

[23] R. P. Dellinger and J. E. Parrillo, Critical Care Medicine: Principles of Diagnosis and Management in the Adult, Elsevier Health Sciences, 2007.

[24] W. Massagram, V. M. Lubecke, and O. Boric-Lubecke, "Microwave non-invasive sensing of respiratory tidal volume," in Proceedings of the Annual International Conference of the IEEE Engineering in Medicine and Biology Society (EMBC '09), pp. 4832-4835, September 2009. 
[25] Y. S. Lee, P. N. Pathirana, C. L. Steinfort, and T. Caelli, "Monitoring and analysis of respiratory patterns using microwave Doppler radar," IEEE Journal of Translational Engineering in Health and Medicine, vol. 2, pp. 1-12, 2014.

[26] P. N. Pathirana, S. C. Herath, and A. V. Savkin, "Multitarget tracking via space transformations using a single frequency continuous wave radar," IEEE Transactions on Signal Processing, vol. 60, no. 10, pp. 5217-5229, 2012.

[27] G. W. Stimson, Introduction to Airborne Radar, Scitech Publishing, San Francisco, 2nd edition, 1998.

[28] A. D. Droitcour, Non-Contact Measurement of Heart and Respiration Rates with a Single-Chip Microwave Doppler Radar, Stanford University, 2006.

[29] R. V. Lenth, "On a form of piecewise linear regression," The American Statistician, vol. 29, no. 3, pp. 116-117, 1975.

[30] R. W. Schafer, "What is a savitzky-golay filter? [Lecture Notes]," IEEE Signal Processing Magazine, vol. 28, no. 4, pp. 111-117, 2011.

[31] T. OHaver, "Interactive fourier filter," September 2006, http:// www.mathworks.com/matlabcentral/fileexchange/12377.

[32] B.-K. Park, S. Yamada, and V. Lubecke, "Measurement method for imbalance factors in direct-conversion quadrature radar systems," IEEE Microwave and Wireless Components Letters, vol. 17, no. 5, pp. 403-405, 2007.

[33] X. Huang, "On transmitter gain/phase imbalance compensation at receiver," IEEE Communications Letters, vol. 4, no. 11, pp. 363$365,2000$.

[34] O. Steila, "Automatic in-phase quadrature balancing," 2006, http://www.qsl.net/iklxpv/dsp/pdf/aiqben.pdf.

[35] S. Salvador and P. Chan, "Toward accurate dynamic time warping in linear time and space," Intelligent Data Analysis, vol. 11, no. 5, pp. 561-580, 2007, http://dl.acm.org/citation.cfm? id= 1367985.1367993.

[36] E. J. Keogh and M. J. Pazzani, "Derivative dynamic time warping," in Proceedings of the 1st SIAM International Conference on Data Mining (SDM '01), 2001.

[37] O. Boric-Lubecke, V. M. Lubecke, I. Mostafanezhad, B.-K. Park, W. Massagram, and B. Jokanovic, "Doppler radar architectures and signal processing for heart rate extraction," Mikrotalasna Revija, pp. 12-17, 2009.

[38] Mosby, Mosby's Medical Dictionary, Elsevier, New York, NY, USA, 2009.

[39] University of Virginia School of Medicine, Asthmaattacks, University of Virginia School of Medicine, 2011, http://www .medicine.virginia.edu/clinical/departments/pediatrics/clinicalservices/tutorials/asthma/attacks.

[40] T. Al-Ani, C. K. Karmakar, A. H. Khandoker, and M. Palaniswami, "Automatic recognition of obstructive sleep apnoea syndrome using power spectral analysis of electrocardiogram and hidden markov models," in Proceedings of the International Conference on Intelligent Sensors, Sensor Networks and Information Processing (ISSNIP '08), pp. 285-290, December 2008 .

[41] B. M. Cappo and D. S. Holmes, "The utility of prolonged respiratory exhalation for reducing physiologial and psychological arousal in non-threatening and threatening situations," Journal of Psychosomatic Research, vol. 28, no. 4, pp. 265-273, 1984.

[42] A. Klintworth, Z. Ajtay, A. Paljunite, S. Szabados, and L. Hejjel, "Heart rate asymmetry follows the inspiration/expiration ratio in healthy volunteers," Physiological Measurement, vol. 33, no. 10, article 1717, 2012.
[43] I. M. Lin, L. Y. Tai, and S. Y. Fan, "Breathing at a rate of 5.5 breaths per minute with equal inhalation-to-exhalation ratio increases heart rate variability," International Journal of Psychophysiology, vol. 91, no. 3, pp. 206-211, 2014. 

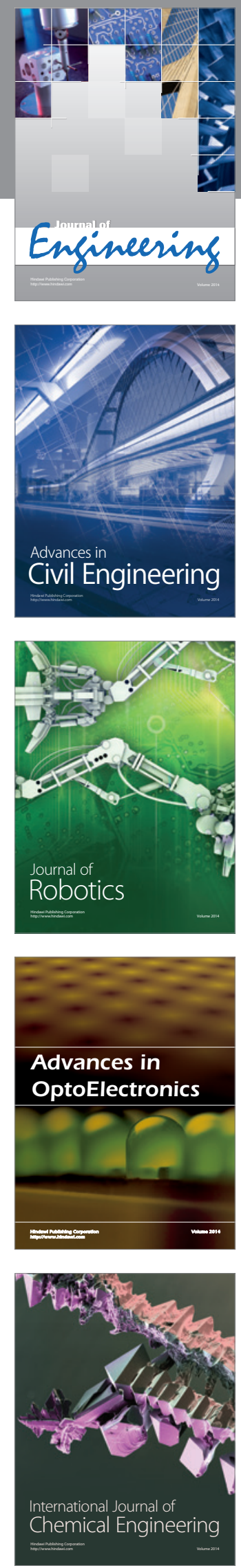

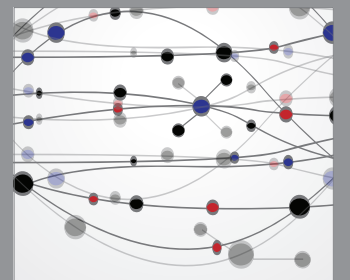

The Scientific World Journal
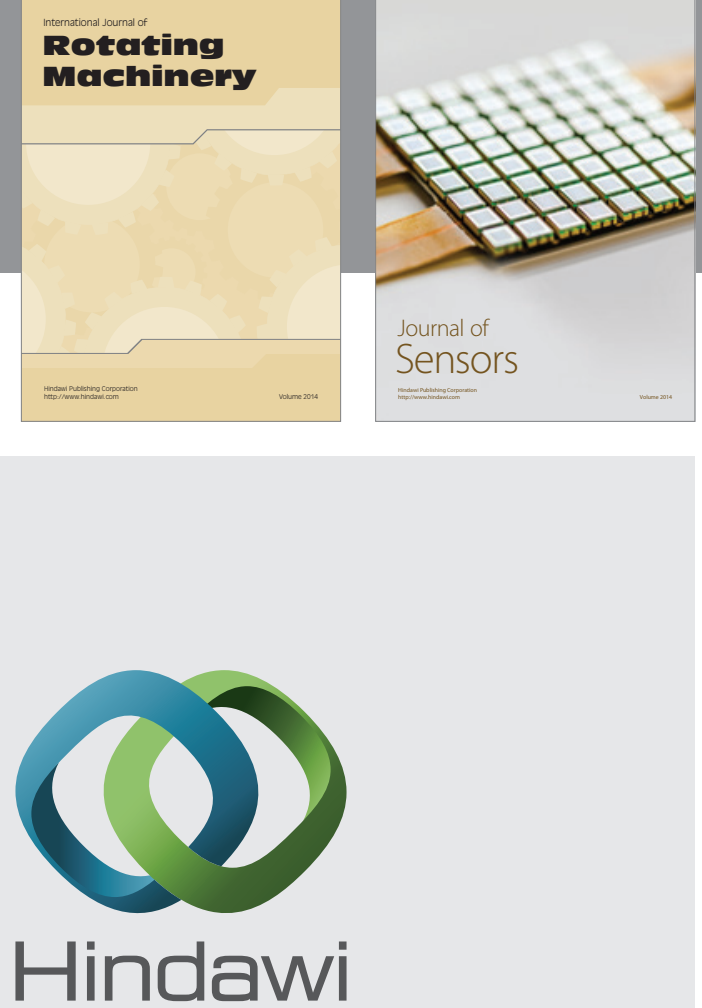

Submit your manuscripts at http://www.hindawi.com
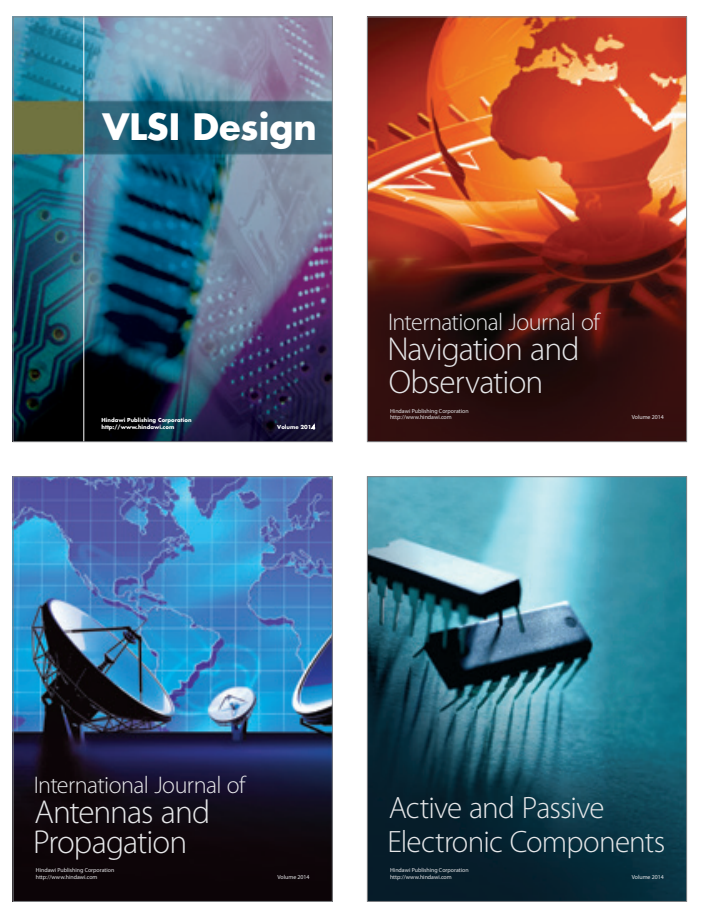
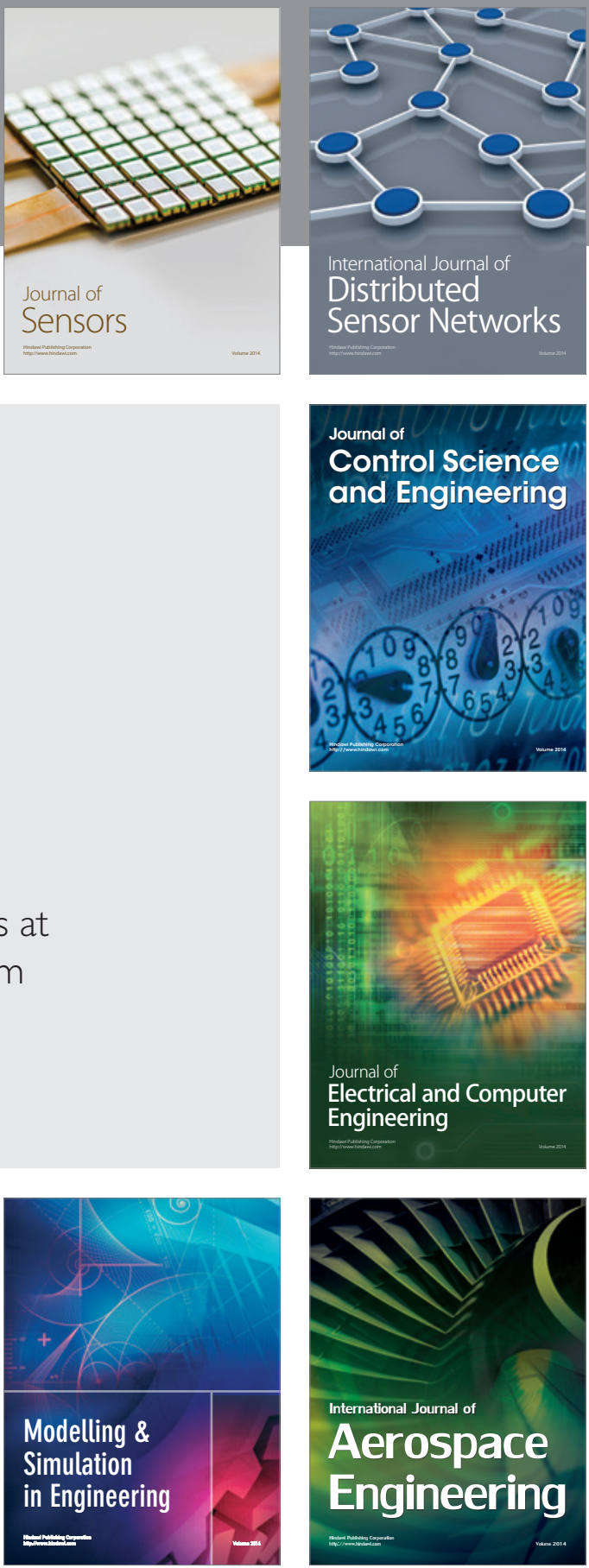

Journal of

Control Science

and Engineering
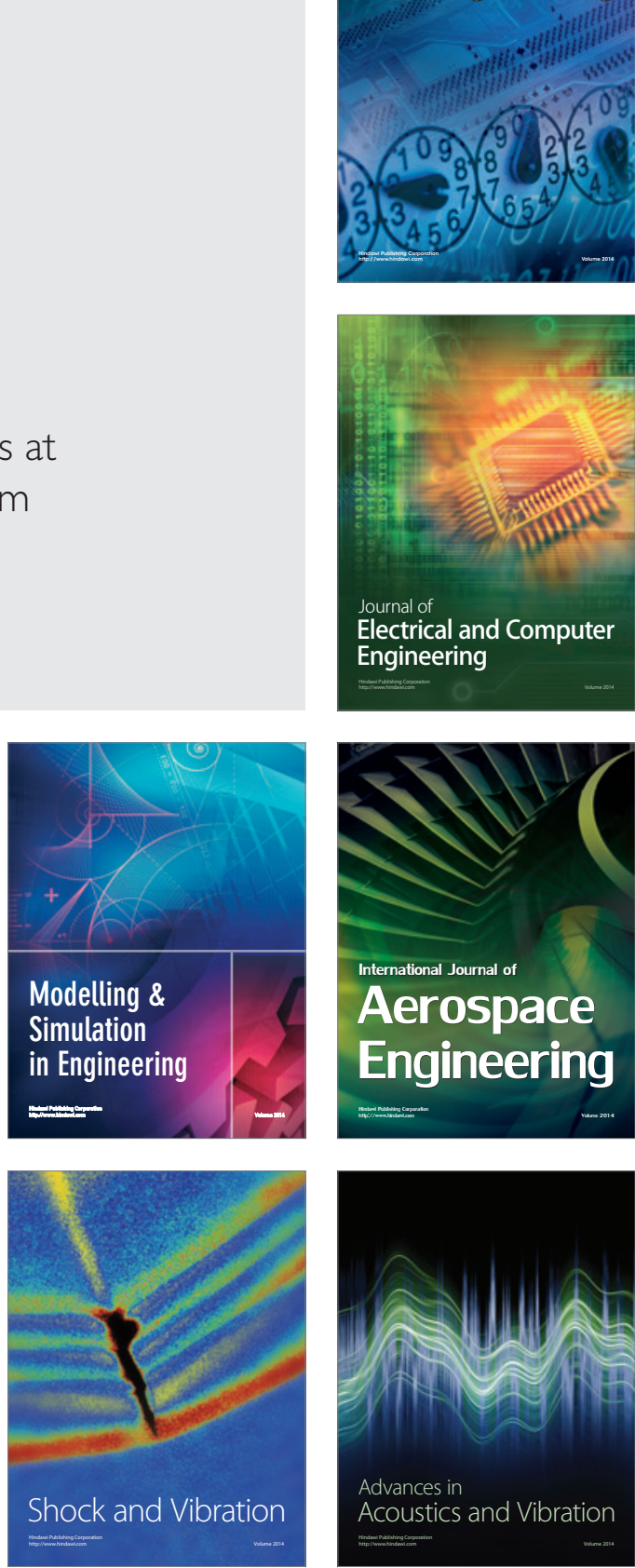